\title{
MicroRNA-451a, microRNA-34a-5p, and microRNA- 221-3p as predictors of response to antidepressant treatment
}

\author{
Wei-Hong Kuang ${ }^{1}$, Zai-Quan Dong ${ }^{1}$, Lian-Tian Tian $^{2}$ and ${\mathrm{Jin} \mathrm{Li}^{1}}^{1}$ \\ ${ }^{1}$ Department of Psychiatry and Mental Health Center, West China Hospital, Sichuan University, Chengdu, China \\ ${ }^{2}$ Research Centre for Public Health and Preventive Medicine, West China School of Public Health, No. 4 West China Teaching \\ Hospital, Sichuan University, Chengdu, China
}

\begin{abstract}
Aberrant expression of microRNAs (miRNAs) has been shown to be involved in early observations of depression. The aim of this study was to determine if serum levels of miRNA-451a, miRNA-34a-5p, and miRNA-221-3p can serve as indicators of disease progression or therapeutic efficacy in depression. We collected data from 84 depressed patients and 78 control volunteers recruited from the medical staff at the West China Hospital. Depression severity was rated using the 24-item Hamilton Depression Scale (HAMD). Serum miRNA-451a, miRNA-34a-5p, and miRNA-221-3p levels were determined in samples from the depressed patients before and 8 weeks after antidepressant treatment as well as in samples from controls. Compared with the controls, the patients had lower miRNA-451a levels, higher miRNA-34a-5p and miRNA-221-3p levels, and increased HAMD scores whether they underwent antidepressant treatment or not. Eight weeks after antidepressant treatment, the patients exhibited increased miRNA-451a levels, decreased miRNA-34a-5p and miRNA-221-3p levels, and reduced HAMD scores. The serum level of miRNA-451a was negatively correlated with HAMD scores of the patients, while the serum levels of miRNA-34a-5p and miRNA-221-3p were positively correlated with HAMD scores whether the patients underwent antidepressant treatment or not. Paroxetine was markedly effective in 50 patients who also displayed an increased level of miRNA-451a but reduced levels of miRNA-34a-5p and miRNA-221-3p. In contrast, paroxetine was moderately effective or ineffective in 34 patients. In conclusion, depressed patients had lower serum miRNA-451a but higher serum miRNA-34a-5p and miRNA-221-3p, and these miRNAs are potential predictors of the efficacy of antidepressants.
\end{abstract}

Key words: MicroRNA-451a; MicroRNA-34a-5p; MicroRNA-221-3p; Depression; Hamilton Depression Scale; Paroxetine

\section{Introduction}

Depression is a serious mental disorder characterized by significant and persistent low mood, retardation, aversion to activity, and repeated suicidal thoughts (1). The incidence and recurrence rates of depression are high (2). According to the World Health Organization, depression affects approximately 154 million people worldwide annually (3). By 2020, depression will become the second leading cause of death and disability (4). The manifestation and pathogenesis of depression is very complex, involving genetic, personality, and social factors (5). The risk factors for depression include changes in expression levels of neurotransmitters, susceptibility of gene polymorphisms, and damaged nerve formation function (6). The treatments for depression primarily include antidepressant drugs and psychological treatment (7). Recent evidence suggests that microRNA (miRNA) may be directly or indirectly involved in the onset and development of depression as well as in the treatment of depression (8).

miRNAs are a class of small RNA molecules that are important in the post-transcriptional regulation of gene expression (9) and can regulate central nervous system functions, including cognitive performance, reward feedback, and circadian rhythm (10). Specific miRNA imbalances may cause a range of neurological disorders, such as Alzheimer's disease and schizophrenia (11). A recent study has shown that abnormal heart and brain tissue could release miRNAs into the circulating blood and cerebrospinal fluid, as evidenced by the presence of significantly abnormal expression of miRNAs in the brain tissue of patients with severe depression who committed suicide (12). miRNA-451a is located in chromosome 17q11.2, and can inhibit the proliferation and growth of cells (13). Research has shown that in cancer tissues, the expression

Correspondence: JinLi: <kwhhknx@163.com> 
of miRNA-451a is down-regulated (14). In addition, miRNA451 was reported to affect the pathogenesis of autism spectrum disorders, and promote neuronal injury in genetically predisposed individuals (15). Studies have shown that serum miRNA-221-3p (16) and miRNA-34a (17) were significantly decreased in depression patients after taking antidepressants.

Therefore, the aim of this study was to compare the miRNA-451a, miRNA-34a-5p, and miRNA-221-3p content in individuals with and without depression, and in patients with depression before and after antidepressant treatment, providing evidence for the early diagnosis and treatment efficacy of depression.

\section{Material and Methods}

\section{Ethics approval}

The study protocol was approved by the Hospital Institutional Review Board of the West China Hospital, Sichuan University. All participants in the study provided written informed consent.

\section{Study participants}

We collected data from 84 patients diagnosed with depression from January 2014 to June 2015 at the West China Hospital, Sichuan University. The inclusion criteria were as follows: 1) meeting diagnostic criteria for depression according to the US Diagnostic and Statistical Manual of Mental Disorders 4th Edition (DSM-IV) (18); 2) no antidepressant therapy for new or previous diagnosis of depression within two weeks prior to enrollment; 3 ) rated $>20$ on the 24-item Hamilton Depression Scale (HAMD) (19); and 4) consent of the patient or family members. The exclusion criteria were as follows: 1) serious physical illness or a history of alcohol or drug abuse; 2) a history of serious heart disease and severe liver or kidney dysfunction; 3) pregnant or lactating women; and 4) a history of manic episodes. Seventy-eight healthy controls were recruited from the same community and during the same time. The controls had HAMD-24 scores of less than 8 points and they had no history of severe traumatic brain disorders or other mental disorders, and no history of suicide attempt. Pregnant or lactating women were not included.

\section{HAMD rating of depression severity}

HAMD is the most widely used scale to assess clinical depression (20), mainly for the depressive symptoms in adult patients. Our study used the 24-item version, while most other studies used a 5-item scale, with 0 to 4 representing "none", "mild", "moderate", "severe", and "very heavy" depression symptom. A few studies have used a 3-item scale, with 0-2 meaning "none", "mild to moderate", and "severe" symptom. A HAMD score of 8 to 20 may indicate depression; a score of 20 to 35 confirms depression; a score above 35 points indicates severe depression. HAMD reduction after antidepressant treatment indicates drug efficacy. The HAMD reduction rate is defined as (baseline score - score after treatment) / baseline score $\times 100 \%$ and is usually used to grade drug efficacy as remarkably effective (HAMD reduction rate $\geqslant 50 \%$ ), effective (HAMD reduction rate $\geqslant 25 \%$ ), or ineffective (HAMD reduction rate $<25 \%$ ). Depressed patients were assessed with HAMD within three days of hospital admission, and the controls were assessed with HAMD after the physical examination. The evaluations were carried out via conversation and observation by two trained attending physicians and scored by two raters independently with a consistency test Kappa of 0.76-0.89. HAMD was also administered to depressed patients after 8 weeks of antidepressant treatment.

\section{Paroxetine treatment regimens}

All depression patients also signed informed consent for treatment with paroxetine (Sino-US Tianjin SmithKline Pharmaceutical Co., Ltd., China). Paroxetine is a new, firstline clinical antidepressant, and its mechanism is to inhibit reuptake of presynaptic 5-HT, resulting in a significant antidepressant effect while being highly safe $(21,22)$. The starting dose of paroxetine was $10 \mathrm{mg} /$ day via oral administration after breakfast. Depending on the patient's condition and the extent of drug resistance, the dose was increased to $20 \mathrm{mg} /$ day in 5 to 7 days and to $30 \mathrm{mg} /$ day before the second weekend (10 to 14 days), with a total duration of 8 weeks. Patient compliance increased after 8 weeks of treatment.

\section{Blood sample collection and processing}

For control samples, $4 \mathrm{~mL}$ of venous blood was collected using an anticoagulant-free disposable vacuum tube under fasting state at the time of physical examination. For depressed patients, $4 \mathrm{~mL}$ of venous blood was collected using an anticoagulant-free disposable vacuum tube under fasting state on the day of HAMD scale evaluation. Another $4 \mathrm{~mL}$ of venous blood was collected from depressed patients after 8 weeks of antidepressant treatment. Blood samples were placed at room temperature to coagulate. After blood coagulation, samples were centrifuged at $1610 \mathrm{~g}$ at room temperature for $10 \mathrm{~min}$. The supernatant was transferred to a microcentrifuge tube and stored at $-80^{\circ} \mathrm{C}$ until quantitative real time polymerase chain reaction ( $\mathrm{qPCR}$ ) analysis. Blood samples with hemolysis were resampled.

\section{qPCR assay}

Serum total RNA was extracted using the frozen serum samples according to kit instructions (Qiagen, USA). Extracted RNA samples were assayed for the 260/280 absorbance value using a UV spectrophotometer, and the RNA concentration was calculated before the samples were stored at $-80^{\circ} \mathrm{C}$ for later use. The reverse transcription of cDNA was conducted following the kit instructions 
(Qiagen). Using the gene sequence database GenBank and the miR database BASE, we designed miRNA-451a, miRNA-34a-5p, and miRNA-221-3p specific reverse transcription primers with a stem-loop structure using Primer 5.0 primer design software (Table 1). Primers were synthesized by Shanghai Sangon Biotech Inc. (China). The qRT-PCR reaction system for miRNA-451a, miRNA-34a$5 p$, and miRNA-221-3p was $20 \mu \mathrm{L}$, including $10 \mu \mathrm{L}$ of SYBR PremixExTaq, $0.4 \mu \mathrm{L}$ of the Forward Primer, $0.4 \mu \mathrm{L}$ of the Reverse Primer, $0.4 \mu \mathrm{L}$ of ROX Reference Dye II, $2 \mu \mathrm{L}$ of the DNA template, and $6.8 \mu \mathrm{L}$ of $\mathrm{dH}_{2} \mathrm{O}$. The reaction conditions were set to $95^{\circ} \mathrm{C}$ for $30 \mathrm{~s}, 95^{\circ} \mathrm{C}$ for $5 \mathrm{~s}$, and $60^{\circ} \mathrm{C}$ for $30 \mathrm{~s}$, for a total of 40 cycles. Using U6 as the internal control, reliability of the results was assessed using the PCR melting curve. The CT value (amplification power curve inflection point) was used to calculate the relative expression of target genes using $2^{-\triangle} \triangle \mathrm{Ct}$ (12).

\section{Statistical analysis}

We used SPSS18.0 statistical software (IBM; USA) for data analysis. Data are reported as means $\pm S D$. Oneway analysis of variance (ANOVA) was used to compare means among multiple groups, and $t$-test was used for comparison between two groups. Correlation among variables was analyzed using the Pearson correlation test. $\mathrm{P}<0.05$ was considered statistically significant.

\section{Results}

\section{Characteristics of patients and controls}

Seventy-eight controls were compared with 84 depressed patients. There were no significant differences between depressed patients and controls with regard to sex, age, education, or family history of depression (all $\mathrm{P}>0.05$ ) (Table 2).

Levels of miRNA-451a, miRNA-34a-5p, and miRNA221-3p, and HAMD scores

qRT-PCR results showed that miRNA-451a expression levels were significantly decreased, and miRNA-34a$5 p$ and miRNA-221-3p expression levels were significantly increased in depressed patients pre- and post-antidepressant treatment (all $\mathrm{P}<0.05$ ) compared with the controls. For depressed patients, miRNA-451a levels increased significantly, while miRNA-34a-5p and miRNA-221-3p levels decreased significantly after 8 weeks of treatment (all $\mathrm{P}<0.05$ ) (Figure $1 \mathrm{~A}$ ).

HAMD evaluation showed that depressed patients had significantly higher HAMD scores than controls (all $P<0.05)$. HAMD scores significantly decreased in patients after 8 weeks of treatment (all $\mathrm{P}<0.05$ ), as shown in Figure 1B.

\section{Correlation between HAMD scores and miRNA levels}

Pearson correlation tests of each miRNA and HAMD score showed that, before and after antidepressant treatment, miRNA-451a level was negatively correlated with HAMD score $(r<0, P<0.05)$, while miRNA-34a-5p and miRNA-221-3p levels were positively correlated with HAMD scores (all $r>0$, all $P<0.05$ ), as shown in Figure 2.

miRNA levels according to paroxetine responses

Based on the HAMD reduction rate, the antidepressant therapy was deemed remarkably effective (HAMD reduction rate $\geqslant 50 \%$ ) for 50 cases, effective (HAMD reduction rate $\geqslant 25 \%$ ) for 25 cases, and ineffective (HAMD reduction rate $<25 \%$ ) for 9 cases. Analysis of miRNA expression levels in different efficacy groups showed no significant difference before treatment (all $P>0.05$ ). However, miRNA-451a was significantly elevated (all $P<0.05$ ), while miRNA-34a-5p and miRNA-221-3p levels declined significantly in the markedly

Table 2. Characteristics of cases and controls.

\begin{tabular}{lccc}
\hline Variable & Controls & Cases & $P$ \\
\hline Number & 78 & 84 & \\
Gender & & & \\
$\quad$ Male & 37 & 30 & 0.152 \\
$\quad$ Female & 41 & 54 & \\
Age (Years) & $35.51 \pm 13.62$ & $39.21 \pm 13.07$ & 0.080 \\
Education $^{\mathrm{a}}$ & $6 / 25 / 20 / 27$ & $7 / 27 / 21 / 29$ & 0.999 \\
Family history & & & \\
$\quad$ No & 62 & 62 & 0.394 \\
Yes & 16 & 22 & \\
\hline
\end{tabular}

Data are reported as means \pm SD or absolute numbers. ${ }^{a}$ Education was categorized into elementary, middle, high school, and college. Statistical analysis was done with the $t$-test or chisquared test.

Table 1. Primer sequences of miRNA-451a, miRNA-34a-5p, miRNA-221-3p, and U6 used in quantitative real time polymerase chain reaction $(\mathrm{qPCR})$.

\begin{tabular}{|c|c|c|}
\hline Gene & Forward & Reverse \\
\hline U6 & 5'-CTCGCTTCGGCAGCACA-3' & 5'-AACGCTTCACGAATTTGCGT-3' \\
\hline miRNA-451a & 5'-ACACTCCAGCTGGGAAACCGTTACCATTACT-3' & 5'-CTGGTGTCGTGGAGTCGGCAA-3' \\
\hline miRNA-34a-5p & 5'-UGGCAGUGUCUUAGCUGGUUGU-3' & 5'-AACCAGCUAAGACACUGCAAUU-3' \\
\hline miRNA-221-3p & 5'-AGCTAAAAAAGCTACATTGTCTGCTGGGTTTCG-3' & 5'-GATCCGAAACCCAGCAGACAATGTAGCTTTTTT-3' \\
\hline
\end{tabular}



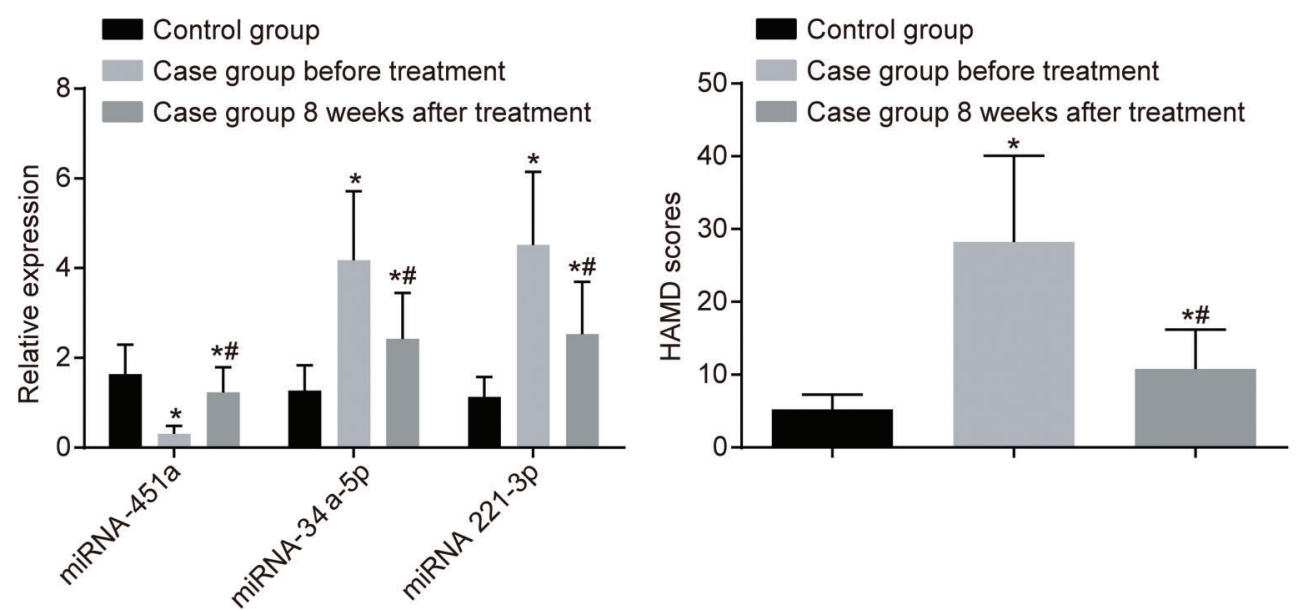

Figure 1. Left panel, eight weeks after paroxetine treatment, miRNA-451a expression was increased while miRNA-34a-5p and miRNA221-3p expressions were decreased. Right panel, eight weeks after paroxetine treatment, the Hamilton Depression Scale (HAMD) scores were decreased. Data are reported as means $\pm S D$. ${ }^{*} \mathrm{P}<0.05$ vs control group; ${ }^{\#} \mathrm{P}<0.05$ vs case group before treatment (ANOVA).
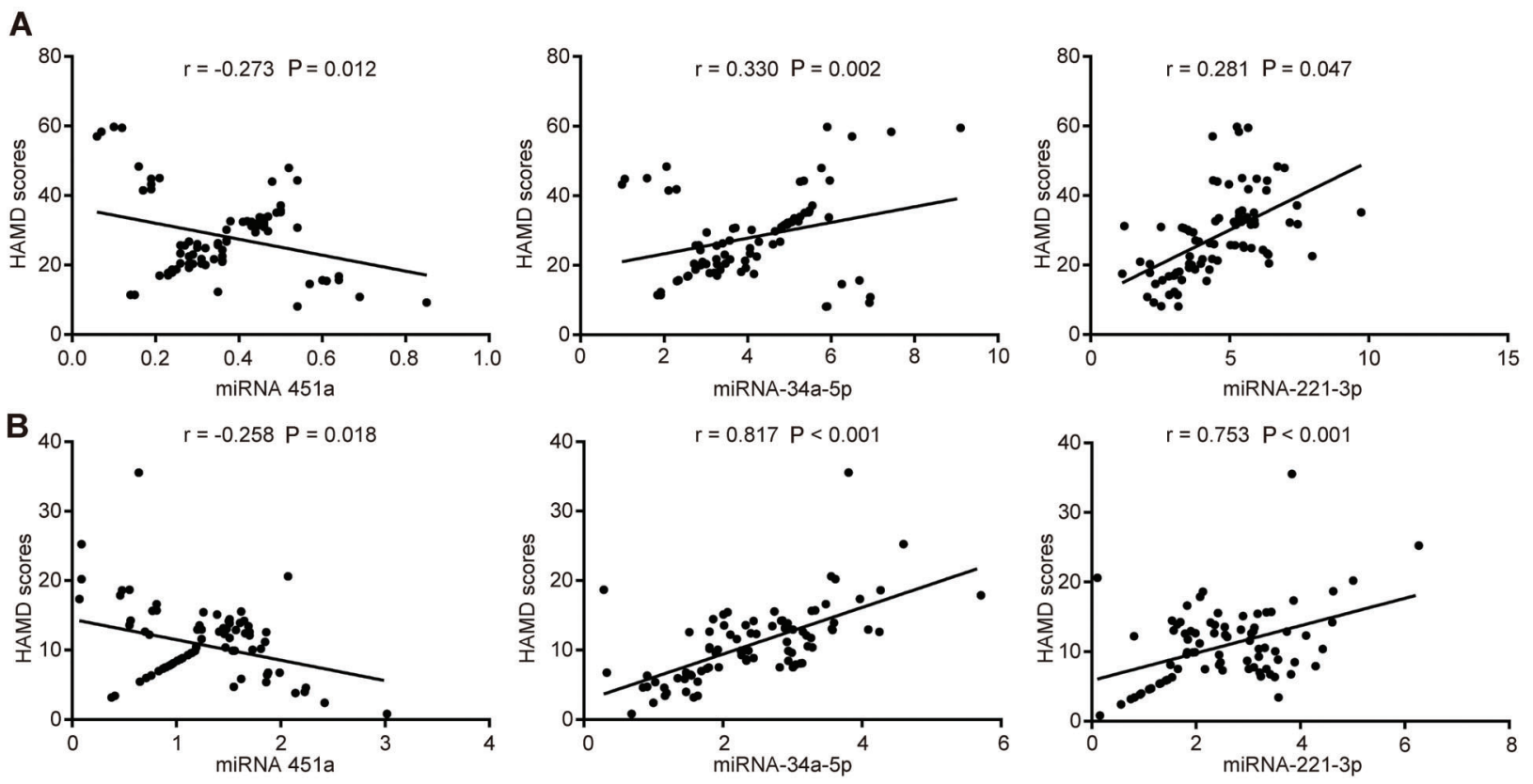

Figure 2. Pearson correlation analysis indicated that miR-451a expression was negatively correlated with the Hamilton Depression Scale (HAMD) scores, while miR-34a-5p and miR-221-3p expressions were positively correlated with HAMD scores before and after paroxetine treatment. Panel $A$, before paroxetine treatment. Panel $B$, after paroxetine treatment. $r<0$ indicates negative correlation while $r>0$ indicates positive correlation.

effective and moderately effective groups (all $P<0.05$ ). There were no significant changes in miRNA-451a, miRNA$34 a-5 p$, and miRNA-221-3p in the ineffective group (all $P>0.05)$. Compared with the markedly effective group, posttreatment miRNA-451a levels were significantly lower $(P<0.05)$, and the miRNA-34a-5p and miRNA-221-3p levels were higher in the moderately effective and ineffective groups (all $\mathrm{P}<0.05$ ). Compared with the moderately effective group, post-treatment miRNA-451a levels were significantly lower $(P<0.05)$, and the miRNA-34a-5p and miRNA-221-3p levels were significantly higher in the ineffective group (all $\mathrm{P}<0.05$ ), as shown in Table 3 . 


\section{Association of miRNA levels with the course of} disease and suicide attempts

Serum miRNA-451a, miRNA-34a-5p, and miRNA-221$3 p$ were not associated with sex, family history, suicidal ideation, first and recurrent depression, or early-onset and late-onset depression before antidepressant treatment (all $P>0.05)$, but were significantly associated with the disease course and history of suicide attempts (all $P<0.05$ ), as shown in Table 4. After treatment, there was no significant association of serum miRNA-451a, miRNA-34a-5p or

Table 3. Relative serum miRNA-451a, miRNA-34a-5p, and miRNA-221-3p levels differed when patients showed different response to antidepressants.

\begin{tabular}{lcc}
\hline miRNA & Before treatment & After treatment \\
\hline Markedly effective group $(n=50$, HAMD reduction rate $\geqslant 50 \%)$ & & \\
miRNA-451a & $0.34 \pm 0.15$ & $1.40 \pm 0.50^{+}$ \\
$\quad$ miRNA-34a-5p & $3.89 \pm 1.19$ & $2.00 \pm 0.73^{+}$ \\
$\quad$ miRNA-221-3p & $4.80 \pm 1.50$ & $2.06 \pm 0.83^{+}$ \\
Effective group $(n=25$, HAMD reduction rate $\geqslant 25 \%)$ & & \\
miRNA-451a & $0.27 \pm 0.18$ & $1.14 \pm 0.57^{+*}$ \\
miRNA-34a-5p & $4.55 \pm 1.48$ & $2.67 \pm 0.99^{+*}$ \\
miRNA-221-3p & $4.25 \pm 1.73$ & $2.81 \pm 1.12^{+*}$ \\
Ineffective group $(n=9$, HAMD reduction rate $<25 \%)$ & & \\
$\quad$ miRNA-451a & $0.29 \pm 0.18$ & $0.53 \pm 0.29^{\star \#}$ \\
miRNA-34a-5p & $4.74 \pm 2.85$ & $4.05 \pm 0.72^{\star \#}$ \\
miRNA-221-3p & $3.73 \pm 1.84$ & $4.38 \pm 0.82^{\star \#}$ \\
\hline
\end{tabular}

Data are reported as means $\pm \mathrm{SD}$. ${ }^{+} \mathrm{P}<0.05$ compared with pre-treatment; ${ }^{*} \mathrm{P}<0.05$ compared with the markedly effective group after treatment; ${ }^{\#} \mathrm{P}<0.05$ compared with the effective group after treatment (ANOVA). miRNA: microRNA; HAMD: 24-item Hamilton Depression Scale.

Table 4. Relationships between relative serum miRNA-451a, miRNA-34a-5p, and miRNA-221-3p levels and suicidal behavior before treatment.

\begin{tabular}{|c|c|c|c|c|c|c|}
\hline & miRNA-451a & $\mathrm{P}$ & miRNA-34a-5p & $P$ & miRNA-221-3p & $P$ \\
\hline Gender & & 0.230 & & 0.173 & & 0.471 \\
\hline Male & $0.28 \pm 0.18$ & & $3.87 \pm 1.79$ & & $4.69 \pm 2.05$ & \\
\hline Female & $0.33 \pm 0.17$ & & $4.35 \pm 1.37$ & & $4.42 \pm 1.36$ & \\
\hline Family history & & 0.674 & & 0.159 & & 0.660 \\
\hline Yes & $0.30 \pm 0.20$ & & $3.78 \pm 1.72$ & & $4.39 \pm 1.91$ & \\
\hline No & $0.32 \pm 0.16$ & & $4.32 \pm 1.46$ & & $4.57 \pm 1.54$ & \\
\hline Suicide attempts & & 0.014 & & $<0.001$ & & 0.0110 \\
\hline Yes & $0.23 \pm 0.16$ & & $5.30 \pm 1.78$ & & $5.35 \pm 1.71$ & \\
\hline No & $0.34 \pm 0.17$ & & $3.85 \pm 1.31$ & & $4.28 \pm 1.54$ & \\
\hline Suicidal ideation & & 0.217 & & 0.358 & & 0.599 \\
\hline Yes & $0.28 \pm 0.16$ & & $3.96 \pm 1.80$ & & $4.39 \pm 1.92$ & \\
\hline No & $0.33 \pm 0.18$ & & $4.29 \pm 1.40$ & & $4.59 \pm 1.48$ & \\
\hline Course of disease & & 0.003 & & 0.012 & & $<0.001$ \\
\hline$\geqslant 5$ years & $0.26 \pm 0.16$ & & $4.56 \pm 1.70$ & & $5.10 \pm 1.67$ & \\
\hline$<5$ years & $0.37 \pm 0.17$ & & $3.72 \pm 1.18$ & & $3.82 \pm 1.27$ & \\
\hline \multicolumn{7}{|l|}{ Type of disease } \\
\hline First & $0.34 \pm 0.17$ & 0.111 & $4.28 \pm 1.26$ & 0.517 & $4.26 \pm 1.33$ & 0.110 \\
\hline Recurrent & $0.28 \pm 0.17$ & & $4.06 \pm 1.82$ & & $4.83 \pm 1.89$ & \\
\hline \multicolumn{7}{|l|}{ Disease onset } \\
\hline Late-onset $(\geqslant 30$ years) & $0.30 \pm 0.18$ & 0.626 & $4.05 \pm 1.78$ & 0.604 & $4.97 \pm 2.18$ & 0.091 \\
\hline Early-onset ( $<30$ years) & $0.32 \pm 0.17$ & & $4.24 \pm 1.43$ & & $4.32 \pm 1.28$ & \\
\hline
\end{tabular}

Data are reported as means $\pm S D(t$-test). miRNA, microRNA. 
miRNA-221-3p with sex, family history, suicidal ideation, first and recurrent depression, early- and late-onset depression, disease course, or history of suicide attempts (all $\mathrm{P}>0.05)$, as shown in Table 5 .

\section{Association of HAMD scores with disease course and suicide attempts}

Pre-treatment HAMD score was not associated with sex, family history, suicidal ideation, first and recurrent depression, or late-onset and early-onset depression (all $P>0.05$ ), but was significantly associated with disease course and history of suicide attempts (all $P<0.05$ ). After 8 weeks of treatment, HAMD scores were not associated with sex, family history, suicidal ideation, first and recurrent depression, late- and early-onset depression, course of disease, or history of suicide attempts (all $\mathrm{P}>0.05$ ), as shown in Table 6.

\section{Discussion}

This study aimed to explore the associations of miRNA-451a, miRNA-34a-5p, and miRNA-221-3p with antidepressant drug efficacy. Our findings suggest that patients with depression had decreased serum miRNA451a levels and increased miRNA-34a-5p and miRNA221-3p levels, which are closely related to the therapeutic efficacy of antidepressant drugs. These miRNAs have the potential to become biomarkers for early diagnosis and therapeutic efficacy for depression.

The results of this study showed that, compared with controls, depressed patients had reduced expression of miRNA-451a but increased miRNA-34a-5p and miRNA221-3p expression levels, which can be reversed with antidepressant treatment. It has been shown that a large number of miRNA are specifically expressed or enriched in the brain or central nervous system, and as a neurological disorder, depression may lead to disordered miRNA expression (23). In addition, the turnover of miRNA in neurons is faster than in other cell types, suggesting that the neuronal miRNA system could result in the rapid adaptation to neuronal activity and be associated with the calpaindependent activation (24). Our findings are consistent with those of Wan et al. (25), who also observed reduced expression of miRNA-451a, and increased expression of miRNA-221-3p in depression patients. Additionally, miRNA451a was demonstrated to work as a candidate biomarker for depression based on the mechanism of action of ketamine (26). It was reported that overexpression of miRNA-34a can lower brain-derived neurotrophic factor (BDNF) expression (27), which is considered one of the major etiologic mechanisms of depression (28). In addition, high expression of miRNA-34a can reduce the expression of the SIRT1 gene (29), which may also contribute to the pathogenesis of depression. Thus, changes in miRNA

Table 5. Relationships between relative serum miRNA-451a, miRNA-34a-5p, and miRNA-221-3p levels and suicidal behavior 8 weeks after treatment.

\begin{tabular}{|c|c|c|c|c|c|c|}
\hline & miRNA-451a & $\mathrm{P}$ & miRNA-34a-5p & $\mathrm{P}$ & miRNA-221-3p & $\mathrm{P}$ \\
\hline Gender & & 0.355 & & 0.612 & & 0.603 \\
\hline Male & $1.15 \pm 0.67$ & & $2.34 \pm 1.19$ & & $2.44 \pm 1.35$ & \\
\hline Female & $1.27 \pm 0.50$ & & $2.46 \pm 0.94$ & & $2.58 \pm 1.07$ & \\
\hline Family history & & 0.832 & & 0.587 & & 0.608 \\
\hline Yes & $1.25 \pm 0.59$ & & $2.32 \pm 1.20$ & & $2.42 \pm 1.36$ & \\
\hline No & $1.22 \pm 0.56$ & & $2.46 \pm 0.97$ & & $2.57 \pm 1.10$ & \\
\hline Suicide attempts & & 0.068 & & 0.46 & & 0.455 \\
\hline Yes & $1.44 \pm 0.80$ & & $2.57 \pm 1.45$ & & $2.71 \pm 1.65$ & \\
\hline No & $1.17 \pm 0.47$ & & $2.37 \pm 0.88$ & & $2.48 \pm 1.00$ & \\
\hline Suicidal ideation & & 0.706 & & 0.077 & & 0.077 \\
\hline Yes & $1.19 \pm 0.63$ & & $2.14 \pm 1.20$ & & $2.21 \pm 1.37$ & \\
\hline No & $1.24 \pm 0.54$ & & $2.56 \pm 0.91$ & & $2.69 \pm 1.04$ & \\
\hline Course of disease & & 0.073 & & 0.084 & & 0.087 \\
\hline$\geqslant 5$ years & $1.33 \pm 0.63$ & & $2.60 \pm 1.15$ & & $2.73 \pm 1.31$ & \\
\hline$<5$ years & $1.11 \pm 0.44$ & & $2.21 \pm 0.83$ & & $2.29 \pm 0.94$ & \\
\hline \multicolumn{7}{|l|}{ Type of disease } \\
\hline First & $1.14 \pm 0.44$ & 0.124 & $2.57 \pm 0.81$ & 0.143 & $2.70 \pm 0.92$ & 0.149 \\
\hline Recurrent & $1.33 \pm 0.67$ & & $2.24 \pm 1.22$ & & $2.33 \pm 1.39$ & \\
\hline \multicolumn{7}{|l|}{ Disease onset } \\
\hline Late-onset $(\geqslant 30$ years $)$ & $1.30 \pm 0.65$ & 0.41 & $2.48 \pm 1.16$ & 0.713 & $2.59 \pm 1.32$ & 0.747 \\
\hline Early-onset ( $<30$ years) & $1.19 \pm 0.52$ & & $2.39 \pm 0.97$ & & $2.50 \pm 1.11$ & \\
\hline
\end{tabular}

Data are reported as means $\pm S D$ (t-test). miRNA, microRNA. 
Table 6. Relationships between HAMD scores and suicidal behavior of patients with depression before and 8 weeks after treatment.

\begin{tabular}{|c|c|c|c|c|}
\hline & Before treatment & $\mathrm{P}$ & 8 weeks after treatment & $P$ \\
\hline Gender & & 0.372 & & 0.289 \\
\hline Male & $29.77 \pm 14.09$ & & $9.92 \pm 5.40$ & \\
\hline Female & $27.34 \pm 10.49$ & & $11.23 \pm 5.39$ & \\
\hline Family history & & 0.486 & & 0.374 \\
\hline Yes & $26.68 \pm 14.11$ & & $11.65 \pm 7.59$ & \\
\hline No & $28.75 \pm 11.05$ & & $10.45 \pm 4.42$ & \\
\hline Suicide attempts & & 0.044 & & 0.238 \\
\hline Yes & $32.93 \pm 14.90$ & & $12.05 \pm 6.44$ & \\
\hline No & $26.82 \pm 10.58$ & & $10.39 \pm 5.05$ & \\
\hline Suicidal ideation & & 0.416 & & 0.217 \\
\hline Yes & $26.71 \pm 13.43$ & & $9.73 \pm 5.63$ & \\
\hline No & $28.96 \pm 11.07$ & & $11.28 \pm 5.26$ & \\
\hline Course of disease & & 0.005 & & 0.155 \\
\hline$\geqslant 5$ years & $31.46 \pm 11.99$ & & $11.53 \pm 5.16$ & \\
\hline$<5$ years & $24.26 \pm 10.60$ & & $9.84 \pm 5.61$ & \\
\hline \multicolumn{5}{|l|}{ Type of disease } \\
\hline First & $27.90 \pm 11.12$ & 0.801 & $11.43 \pm 5.15$ & 0.288 \\
\hline Recurrent & $28.56 \pm 12.83$ & & $10.00 \pm 5.64$ & \\
\hline \multicolumn{5}{|l|}{ Disease onset } \\
\hline Late-onset ( $\geqslant 30$ years) & $31.02 \pm 13.30$ & 0.148 & $10.54 \pm 5.28$ & 0.798 \\
\hline Early-onset ( $<30$ years) & $26.95 \pm 11.07$ & & $10.87 \pm 5.50$ & \\
\hline
\end{tabular}

Data are reported as means \pm SD ( $t$-test). HAMD, 24-item Hamilton Depression Scale.

expression could affect the expression of many genes related to neural activity in the brain, leading to the onset and development of depression.

Our results also suggested that 8 weeks of antidepressant treatment could significantly increase miRNA-451a expression, decrease miRNA-34a-5p and miRNA-221$3 p$ expression, and decrease HAMD scores. Previous studies showed that antidepressants can lower miRNA221-3p (16) and miRNA-34a-5p (17) levels, which is consistent with the results of our study. Furthermore, comparison among groups with different levels of treatment efficacy showed that the increase in miRNA-451a levels and the decrease in miRNA-34a-5p and miRNA-221-3p levels are positively associated with higher antidepressant efficacy. It was reported that miRNA-221 in serum of patients with major depressive disorder was downregulated after treatment, indicating that antidepressant treatment has a normalizing effect on the circulating miRNA levels (30). Moreover, miRNA-34c-5p has been previously demonstrated to affect the basic mechanisms of brain neuroplasticity and stress response (31). A study of the relationship between early treatment outcome and suicidal ideation in 705 cases of hospitalized depression patients using HAMD score showed that the incidence of suicide ideation was 3-5 times higher in patients who had low treatment efficacy than in patients who had high treatment efficacy and that early treatment efficacy significantly reduced pessimism (32). Therefore, it is critical to evaluate treatment efficacy in a timely manner. Based on the above findings, miRNA-451a, miRNA-34a-5p, and miRNA-221-3p could become potential markers for early diagnosis and therapeutic efficacy.

The results also showed that serum miRNA-451a, miRNA-34a-5p, and miRNA-221-3p expression was closely associated with the disease course and suicide attempts. Patients with $\geqslant 5$ years of depression have different miRNA-451a, miRNA-34a-5p, and miRNA-221$3 p$ expression than those diagnosed for fewer than 5 years. A previous study has shown that a longer course of depression is associated with worse cognitive dysfunction, and loss of interest, self-efficacy, and awareness (33). Since miRNA may regulate central nervous system functions such as cognitive function and reward feedback, we hypothesized that with the extension of disease course, changes in miRNA-451a, miRNA-34a-5p, and miRNA-221-3p expression may cause the deterioration of the central nervous system. Studies have shown that reduced BDNF plays an important role in depression and suicidal behavior (34). Kim et al. (35) and Lee et al. (36) found that patients with suicidal behavior have lower plasma BDNF than those without suicidal behavior. As previously mentioned, changes in miRNA expression affects the expression of a number of genes related to neural activity in the brain, and so we hypothesize that the low expression 
of BDNF in patients with a history of suicide attempts is caused by disordered expression of miRNA-451a, miRNA$34 a-5 p$, and miRNA-221-3p. Our study found no significant association of serum expression of these miRNAs with suicidal ideation, but there was a significant association with suicide attempts, suggesting the abnormal expression of serum miRNA is more likely to contribute to suicide behavior rather than suicide ideation.

There are several limitations in our study. First, the sample size was relatively small; second, some clinical characteristics and medical history that may influence the outcome were not well matched among all enrolled depression patients; third, the miRNA quantification platforms and sample type need further standardization; fourth, although paroxetine is a new, first-line clinical antidepressant with considerable antidepressant effect and high safety, other antidepressants should be assessed on the same correlations with serum miRNA-451a, miRNA-34a-5p, and miRNA-221-3p levels. Due to these restrictions, the use of serum circulating miRNA-451a, miRNA-34a-5p, and miRNA-221-3p as predictors for assessing antidepressant treatment requires further investigation.

In conclusion, depression patients have reduced serum miRNA-451a levels but increased miRNA-34a-5p and miRNA-221-3p expression levels. The expression levels of these miRNAs are closely related to the efficacy of antidepressant drugs.

\section{Acknowledgments}

This study was supported by National Natural Science Foundation of China (Grant No. 81621003) and Sichuan Science and Technology Plan Program (Grant No. 2015 JY0072).

\section{References}

1. Belmaker RH, Agam G. Major depressive disorder. N Engl J Med 2008; 358: 55-68, doi: 10.1056/NEJMra073096.

2. Kessler RC, Bromet EJ. The epidemiology of depression across cultures. Annu Rev Public Health 2013; 34: 119-138, doi: 10.1146/annurev-publhealth-031912-114409.

3. Greenhalgh T. WHO/WONCA report - Integrating Mental Health in Primary Care: A Global Perspective. London J Prim Care (Abingdon) 2009; 2: 81-82.

4. Dennis CL, Dowswell T. Interventions (other than pharmacological, psychosocial or psychological) for treating antenatal depression. Cochrane Database Syst Rev 2013; 7: CD006795, doi: 10.1002/14651858.CD006795.pub3.

5. Krishnan V, Nestler EJ. The molecular neurobiology of depression. Nature 2008; 455: 894-902, doi: $10.1038 /$ nature 07455.

6. Drevets WC, Price JL, Furey ML. Brain structural and functional abnormalities in mood disorders: implications for neurocircuitry models of depression. Brain Struct Funct 2008; 213: 93-118, doi: 10.1007/s00429-008-0189-x.

7. Jin M. Diagnosing, Screening, and Monitoring Depression in the Elderly: A Review of Guidelines. Ottawa $(O N)$ : Canadian Agency for Drugs and Technologies in Health 2015; 381.

8. Dwivedi Y. Emerging role of microRNAs in major depressive disorder: diagnosis and therapeutic implications. Dialogues Clin Neurosci 2014; 16: 43-61.

9. Friedman RC, Farh KK, Burge CB, Bartel DP. Most mammalian mRNAs are conserved targets of microRNAs. Genome Res 2009; 19: 92-105, doi: 10.1101/gr.082701.108.

10. Cheng HY, Papp JW, Varlamova O, Dziema H, Russell B, Curfman JP, et al. microRNA modulation of circadian-clock period and entrainment. Neuron 2007; 54: 813-829, doi: 10.1016/j.neuron.2007.05.017.

11. Beveridge NJ, Cairns MJ. MicroRNA dysregulation in schizophrenia. Neurobiol Dis 2012; 46: 263-271, doi: 10.1016/j.nbd.2011.12.029.

12. Krishnadas R, Cavanagh J. Depression: an inflammatory illness? J Neurol Neurosurg Psychiatry 2012; 83: 495-502, doi: 10.1136/jnnp-2011-301779.
13. Liu Z, Miao T, Feng T, Jiang Z, Li M, Zhou L, et al. miR--451a Inhibited Cell Proliferation and Enhanced Tamoxifen Sensitive in Breast Cancer via Macrophage Migration Inhibitory Factor. Biomed Res Int 2015; 2015: 207684, doi: 10.1155/ 2015/207684.

14. Fukumoto I, Kinoshita T, Hanazawa T, Kikkawa N, Chiyomaru $\mathrm{T}$, Enokida $\mathrm{H}$, et al. Identification of tumour suppressive microRNA-451a in hypopharyngeal squamous cell carcinoma based on microRNA expression signature. $\mathrm{Br} \mathrm{J}$ Cancer 2014; 111: 386-394, doi: 10.1038/bjc.2014.293.

15. Pagan C, Goubran-Botros H, Delorme R, Benabou M, Lemiere N, Murray K, et al. Disruption of melatonin synthesis is associated with impaired 14-3-3 and miR-451 levels in patients with autism spectrum disorders. Sci Rep 2017; 7: 2096, doi: 10.1038/s41598-017-02152-x.

16. Oved K, Morag A, Pasmanik-Chor M, Rehavi M, Shomron N, Gurwitz D. Genome-wide expression profiling of human lymphoblastoid cell lines implicates integrin beta- 3 in the mode of action of antidepressants. Transl Psychiatry 2013; 3: e313, doi: 10.1038/tp.2013.86.

17. Liu BB, Luo L, Liu XL, Geng D, Liu Q, Yi LT. 7-Chlorokynurenic acid (7-CTKA) produces rapid antidepressant-like effects: through regulating hippocampal microRNA expressions involved in TrkB-ERK/Akt signaling pathways in mice exposed to chronic unpredictable mild stress. Psychopharmacology (Berl) 2015; 232: 541-550, doi: 10.1007/ s00213-014-3690-3.

18. Cooper J. Diagnostic and Statistical Manual of Mental Disorders (4th Edition): DSM-IV[J]. British Journal of Psychiatry 2001; 179:97-98, doi: 10.1192/bjp.179.1.85-a.

19. Bech $P$. The responsiveness of the different versions of the Hamilton Depression Scale. World Psychiatry 2015; 14: 309-310, doi: 10.1002/wps.20248.

20. Paykel ES. Use of the Hamilton Depression Scale in general practice. Psychopharmacol Ser 1990; 9: 40-47, doi: 10.1007/ 978-3-642-75373-2_6.

21. Yoshimura R, Kishi T, Hori H, Ikenouchi-Sugita A, Katsuki A, Umene-Nakano W, et al. Comparison of the efficacy between paroxetine and sertraline augmented with aripiprazole in 
patients with refractory major depressive disorder. Prog Neuropsychopharmacol Biol Psychiatry 2012; 39: 355-357, doi: 10.1016/j.pnpbp.2012.07.008.

22. Boulenger JP, Hermes A, Huusom AK, Weiller E. Baseline anxiety effect on outcome of SSRI treatment in patients with severe depression: escitalopram vs paroxetine. Curr Med Res Opin 2010; 26: 605-614, doi: 10.1185/03007990903482467.

23. Dwivedi Y. Evidence demonstrating role of microRNAs in the etiopathology of major depression. J Chem Neuroanat 2011; 42: 142-156, doi: 10.1016/j.jchemneu.2011.04.002.

24. Mouillet-Richard S, Baudry A, Launay JM, Kellermann O. MicroRNAs and depression. Neurobiol Dis 2012; 46: 272278, doi: 10.1016/j.nbd.2011.12.035.

25. Wan $Y$, Liu $Y$, Wang $X$, Wu J, Liu $K$, Zhou J, et al. Identification of differential microRNAs in cerebrospinal fluid and serum of patients with major depressive disorder. PLoS One 2015; 10: e0121975, doi: 10.1371/journal.pone. 0121975.

26. Camkurt MA, Acar S, Coskun S, Gunes M, Gunes S, Yilmaz MF, et al. Comparison of plasma MicroRNA levels in drug naive, first episode depressed patients and healthy controls. J Psychiatr Res 2015; 69: 67-71, doi: 10.1016/j.jpsychires. 2015.07.023.

27. Wibrand K, Pai B, Siripornmongcolchai T, Bittins $M$, Berentsen $B$, Ofte $M L$, et al. MicroRNA regulation of the synaptic plasticity-related gene Arc. PLoS One 2012; 7: e41688, doi: 10.1371/journal.pone.0041688.

28. Ray MT, Shannon Weickert C, Webster MJ. Decreased BDNF and TrkB mRNA expression in multiple cortical areas of patients with schizophrenia and mood disorders. Transl Psychiatry 2014; 4: e389, doi: 10.1038/tp.2014.26.

29. Li L, Yuan L, Luo J, Gao J, Guo J, Xie X. MiR-34a inhibits proliferation and migration of breast cancer through down-regulation of Bcl-2 and SIRT1. Clin Exp Med 2013; 13: 109-117, doi: 10.1007/s10238-012-0186-5.

30. Enatescu VR, Papava I, Enatescu I, Antonescu M, Anghel A, Seclaman E, et al. Circulating plasma micro RNAs in patients with major depressive disorder treated with antidepressants: a pilot study. Psychiatry Investig 2016; 13: 549-557, doi: 10.4306/pi.2016.13.5.549.

31. Bocchio-Chiavetto L, Maffioletti E, Bettinsoli P, Giovannini C, Bignotti $S$, Tardito $D$, et al. Blood microRNA changes in depressed patients during antidepressant treatment. Eur Neuropsychopharmacol 2013; 23: 602-611, doi: 10.1016/ j.euroneuro.2012.06.013.

32. Seemuller F, Schennach-Wolff R, Obermeier M, Henkel V, Moller HJ, Riedel M. Does early improvement in major depression protect against treatment emergent suicidal ideation? J Affect Disord 2010; 124: 183-186, doi: 10.1016/ j.jad.2009.10.010.

33. McClintock SM, Husain MM, Greer TL, Cullum CM. Association between depression severity and neurocognitive function in major depressive disorder: a review and synthesis. Neuropsychology 2010; 24: 9-34, doi: 10.1037/ a0017336.

34. Dwivedi Y. Brain-derived neurotrophic factor and suicide pathogenesis. Ann Med 2010; 42: 87-96, doi: 10.3109/ 07853890903485730.

35. Kim YK, Lee HP, Won SD, Park EY, Lee HY, Lee BH, et al. Low plasma BDNF is associated with suicidal behavior in major depression. Prog Neuropsychopharmacol Biol Psychiatry 2007; 31: 78-85, doi: 10.1016/j.pnpbp.2006. 06.024.

36. Lee BH, Kim H, Park SH, Kim YK. Decreased plasma BDNF level in depressive patients. J Affect Disord 2007; 101: 239244, doi: 10.1016/j.jad.2006.11.005. 